\title{
Stellar Variabilities: Challenges for the Detection and Characterization of Exoplanets
}

\author{
I. Boisse ${ }^{1}$, X. Dumusque ${ }^{1,2}$, N. C. Santos ${ }^{1,3}$, M. Oshagh ${ }^{1,3}$, X. Bonfils ${ }^{4}$, \\ M. Montalto ${ }^{1}$, G. Boué ${ }^{1}$ and C. Lovis ${ }^{2}$ \\ ${ }^{1}$ Centro de Astrofísica, Universidade do Porto, Rua das Estrelas, 4150-762 Porto, Portugal \\ email: Isabelle.Boisse@astro.up.pt \\ ${ }^{2}$ Observatoire de Genève, Université de Genève, 51 Ch. des Maillettes, 1290 Sauverny, \\ Switzerland \\ ${ }^{3}$ Departamento de Física e Astronomia, Faculdade de Ciências, Universidade do Porto, \\ 4169-007 Porto, Portugal \\ ${ }^{4}$ UJF-Grenoble1/CNRS-INSU, Institut de Planétologie et d'Astrophysique de Grenoble, UMR \\ 5274, Grenoble, F-38041, France
}

\begin{abstract}
The photometric and RV techniques, although extremely efficient to detect and characterize planets, are, however, indirect techniques (as well as astrometry). Phenomena such as stellar pulsation, inhomogeneous convection, spots or magnetic cycles can prevent us from finding planets or they might degrade the parameters estimation. We will consider the challenges related to the knowledge of stellar activity for the next decade: detect telluric planets in the habitable zone of their stars (from $\mathrm{G}$ to $\mathrm{M}$ dwarfs), understand the activity in the low-mass end of $\mathrm{M}$ dwarf (on which will focus future near-infrared high-resolution spectrograph like SPIRou or CARMENES), limitation to the process of summing several transit observations (in order to characterize the atmospheric components) due to the variability of stellar activity (from the ground or with Spitzer or JWST), as well as the methods proposed and used to overcome this issue.
\end{abstract}

Keywords. Techniques: radial velocities, photometry, Stars: activity, Dynamo, Planetary system

\section{Introduction}

Up to now, more than 900 planets have been discovered, most of them using the radial-velocity $(\mathrm{RV})$ technique. However, this efficient method is indirect and so are the photometric transit and astrometry techniques. One of the problems is that RV variations can in some cases be caused by other mechanisms that are not related to the presence of low-mass companions. Even if RV surveys could reject very active targets, all stars present variability at different levels (from few $\mathrm{cms}^{-1}$ to hundreds of $\mathrm{ms}^{-1}$ ). Moreover, RV is mainly needed to establish the planetary nature and measure the mass of a transiting candidate (and stellar activity is less determinant for transit detection).

RV values are derived from the determination of position in wavelength of thousands of spectral lines. Unfortunately, not only is the measurement sensitive to Doppler shift due to gravitationally bounded companion, it is also sensitive to line profile deformations. Especially, the appearance and disappearance of spots from the observer view due to the stellar rotation, or change of the convection pattern with magnetic cycle induces a distortion of the line that changes with time. The jitter that results in RV may hamper planet discovery or degrade the orbital parameters estimation. They may also give false 
candidates, if they produce a stable periodic signal (e.g. Queloz et al. 2001, Huélamo et al. 2008, Figueira et al. 2010). A fundamental work is then needed to understand all phenomena caused by stellar variability, and to characterize their impact on RV and photometry to be able to distinguish between these and real planetary signatures.

\section{Overcome the stellar variability}

\section{Different frequencies: observational strategy}

Phenomena such as stellar pulsation or inhomogeneous convection could be averaged by specific observational strategy (Dumusque et al. 2011a). Because the typical timescale of those oscillations is less than fifteen minutes, an exposure time of fifteen minutes or larger reduces greatly the oscillation noise. In the same way, the timescale of granulation is less than 30 hours, therefore taking several measurements per night allows a reduction of the RV contribution of granulation. These methods have already been used (Pepe et al. 2011) and showed good efficiency (e.g. Santos et al. 2004) to use averaged asteroseismologic data to detect one of the first low-mass planet. Moreover, Dumusque et al. (2011b) showed that the noise induced by activity-related stellar spot groups could be average by several measurements per month when we are searching for planets with periods of hundreds of days (i.e. planets in the habitable zone of $\mathrm{G}-\mathrm{K}$ dwarfs). When the frequency of the searched object is different to the noise, the stellar variability should be averaged thanks to a clever observational strategy.

\section{Comparable frequencies: diagnostics and models}

When the frequencies are comparable, we need diagnostics (observations) and models (simulations) in order to characterize and disentangle between stellar variability and planetary signals. In the following, we focus on stellar activity, mainly dark spots and bright plages on stellar surface, and on magnetic cycle. These phenomenon are assumed to be part of the stellar dynamo process that generates stellar magnetic field.

To identify stellar activity, we can monitor the bisector span (BIS), the width and the depth of the mean line of the spectrum, and also the chromospheric emission ratio, $\log \mathrm{R}_{\mathrm{H} K}^{\prime}$ (calculated from the CaII H \& K lines) as well as the other lines sensitive to active features (e.g. $\mathrm{H} \alpha$, HeI, NaI). However the behavior of these parameters is not yet properly understood as they depend on stellar parameters (Desort et al. 2007, Boisse et al. 2011, Boisse et al. 2012b). For example, Bonfils et al. (2007) had to compare RV to index derived from $\mathrm{H} \alpha$ and CaII $\mathrm{H} \& \mathrm{~K}$, because the BIS becomes inefficient as an indicator of activity when the star rotates too slow. Nevertheless, Boisse et al. (2009), monitoring these indicators on HD189733 during two rotational period of the star were able to demonstrate the main phenomenon on the photosphere. They identified a dark spot associated with a bright plage that dominated the RV and the photometric signals. Until now, these SOPHIE/MOST observations are the references as simultaneous highaccuracy RV and high-precision photometry (Lanza et al. 2011).

$\mathrm{SOAP} \dagger$ is an online software that simulates the effect of stellar spots and plages on RV and photometry (Boisse et al. 2012b). This numerical model centers the visible stellar disk on a grid of several tens of thousands of resolution elements. The typical line of the emerging spectrum of the star is modeled by a Gaussian for each grid cell (equivalent to the spectrum cross-correlation function that can be seen as a weighted average of all the spectral lines convolved with a rectangular function). Each Gaussian (in a given stellar position) is Doppler-shifted according to the projected rotational velocity and

$\dagger$ The tool is available at http://www.astro.up.pt/soap 
weighted by a linear limb-darkening law. SOAP calculates the position of the surface inhomogeneities defined by their latitudes, longitudes, and sizes. The cells inside the spots are modeled by the same Gaussian as for the stellar disk, but are weighted by their brightness. The code then removes (for dark spot) or adds (for bright plage) the Gaussian and flux of the inhomogeneities to those of the non-spotted star. Finally, SOAP delivers the integrated spectral line, the flux, the RV, and the bisector span as a function of the stellar rotational phase. The code allows characterizing the variations of the RV, BIS, and photometric amplitude as a function of the main variables: projected stellar rotational velocity, filling factor of the spot, resolution of the spectrograph, linear limb-darkening coefficient, latitude of the spot, and inclination of the star.

Boisse et al. (2011) used this tool to develop a method to correct from activity thanks to a harmonic decomposition of the period of the stellar rotation. This was part of an effort to correct stellar activity in RV measurements. The first technique used by e.g. Melo et al. (2007) and Boisse et al. (2009) was to subtract the RV-BIS correlation to the data. The case of CoRoT-7 where the signal of the planets is embedded in the stellar activity noise leads to other techniques such as pre-whitening (e.g. Queloz et al. 2009), Fourier analysis (Hatzes et al. 2010) or self-consistent high-pass filter (Ferraz-Mello et al. 2011). However all these techniques have weaknesses, and a lot of data or indices are often needed to well-understand the phenomenon.

It is only recently that the discoveries of planets with orbital periods reaching the range where stellar magnetic cycles have been observed (from 2.5 to 25 years, Baliunas et al. 1995), have been achievable. A magnetic cycle could induce RV variations with the periodic modification of the number of spots and plages on the stellar photosphere (as observed on the Sun on a 11-year period), related to changes in the convection pattern and/or other mechanisms such as meridional flows, owing to the magnetic field created by dynamo. The $\log \mathrm{R}_{\mathrm{H} K}$ index computed from the CaII H\&K lines is sensitive to the presence of plages in the stellar chromosphere and is a reliable means of monitoring the magnetic cycle. Dedicated RV observations of stars with known magnetic cycles (Santos et al. 2010, Gomes da Silva et al. 2012) have measured weak correlations between active lines indices (CaII H\&K, $\mathrm{H} \alpha, \mathrm{NaI}$ ) and $\mathrm{RV}$, as w ell as in the parameters of the CCF. However, these studies have been limited by a narrow range of spectral types, respectively, early-K and early-M dwarfs. On the other hand, high-precision stabilized fiber-fed spectrographs that observe in the visible such as HARPS or SOPHIE can accurately measure the flux in the CaII H\&K lines. They can monitor with high precision the variation with time in the $\log _{\mathrm{H} K}$ index. While searching for planets, HARPS RV measurements have revealed stellar magnetic cycles (Moutou et al. 2011, Ségransan et al. 2011, Dumusque et al. 2011c). Lovis et al. (2011) used the HARPS sample to identify activity cycles and derive relations between the RV and CCF parameter variations as a function of the $R_{\mathrm{H} K}^{\prime}$ variability. These relations depend on the stellar effective temperature and could be used to estimate the RV jitter produced by a magnetic cycle. RV detection of Jupiter-like planets on long periods needs to be careful with this effect (e.g. Wright et al. 2008, Boisse et al. 2012a ).

\section{Futur and SOAP developments}

\section{SOAP improvements}

$\mathrm{SOAP}-\mathrm{T} \dagger$ is an improved version of SOAP that includes the impact of a transiting planet in RV and photometry (Oshagh et al. 2013). A planet transiting its host-star could overlap with active zones on the stellar surface. It will generate anomalies (bumps) inside the

$\dagger$ The tool is available at http://www.astro.up.pt/resources/soap-t/ 
transit light-curve (Pont et al. 2007, Rabus et al. 2009). The evolution of these anomalies could have been tracked had long-term and continuous observations been accessible (e.g. CoRoT and Kepler). It could allow to determine the spin-orbit angle, the inclination of the stellar spin axis, and the configuration of the spots on the stellar surface (e.g. Nutzman et al. 2011, Désert et al. 2011, Sanchis-Ojeda et al. 2011, Sanchis-Ojeda \& Winn 2011).

In future, we plan to add a spectral capability to the tools. While the tools simulate the photometric effect of spots, they can also block the convection pattern, leading to a general red-shifted light. Moreover, not only are spots and plages areas darker or brighter, they also have a different temperature than the stellar photosphere. This wavelength dependency must be taken into account because despite most of the accurate spectrographs being now in the optical, soon near infrared instruments will be available for wavelength domains where the amplitude of RV variations due to activity are supposed to be smaller (e.g. Huélamo et al. 2008, Czesla et al. 2009).

\section{Transit determination}

When a planet transits in front of its star, its radius is determined as a function of the occulted flux. When this planet is also characterized by RV, which yields its mass, a measure of its mean density is obtained, which is the first step in understanding the internal characterization of exoplanets. But the stellar inhomogeneities may have impacts on the radius determination derived from transit detections. One of the impacts is that they hampers a correct determination of the radius. The correct value of a planet's radius will be crucial for determining atmospheric components that are derived from the radius variations as a function of wavelength (e.g. Czesla et al. 2009, Berta et al. 2011, Knutson et al. 2011, Désert et al. 2011, Sing et al. 2011, Pont et al. 2013).

\section{Focus on low-mass stars}

M-dwarfs present several advantages in the search and characterization of extrasolar planets compared to the FGK stars. First, their lower masses $\left(0.07<M_{\star}<0.6 \mathrm{M}_{\odot}\right)$ implies that small-mass planets around them are easier to detect (i.e. the semi-amplitude of the effect of a planet in $\mathrm{RV} \alpha M_{\star}^{-2 / 3}$ is larger around low-mas stars). Their smaller radii cause the transit depth (that depends on $M_{\star}^{-2}$ ) to be larger. Their stellar effective temperatures are lower and therefore their habitable zones are in short period orbits. Another advantage of low-mass stars is that they are the most numerous in the solar neighborhood. That is why many of the current and future observational project have focused on these targets. To highlight a few, we mentioned for example, MEarth project that is currently searching for transits around $\mathrm{M}$ stars from the ground (Charbonneau et al. 2008), the high-accuracy spectrographs currently with CRIRES (Bean et al. 2010), HARPS (Bonfils et al. 2013), CARMENES (Quirrenbach et al. 2012) and SPIRou (Artigau et al. 2011).

One issue with M-dwarfs is that they are faint and as a result there are several problems in determining their parameters accurately. Also, a great number of these stars are active (mainly beyond spectral type M4, West et al. 2004). As mentioned previously, one solution to this problem can come from the lower impact of spots in the nIR compared to the visible. The amplitude of the effect in RV depends on the difference of flux emitted in the wavelength domain between the photosphere and the spot (e.g. Martin et al. 2006, Prato et al. 2008). Another solution can come from the combine observation with spectropolarimetry, sensitive to the magnetic field. 


\section{Acknowledgements}

IB, MO, NCS, MM, and GB acknowledge the support of the European Research Council/European Community under the FP7 through a Starting Grant, as well from Fundaccao para a Ciência e a Tecnologia (FCT), Portugal, through SFRH/BPD/81084/2011 and the project PTDC/CTE-AST/098528/2008.

\section{References}

Artigau, E., Donati, J.-F., \& Delfosse, X. 2011, ASPC, 448, 771

Baliunas, S., Donahue, R., Soon, W., et al. 1995, ApJ, 438, 269

Bean, J., Seifahrt, A., Hartman, H., et al. 2010, ApJ, 713, 410

Berta, Z., Charbonneau, D., Bean J., et al. 2011, ApJ, 736, 12

Boisse, I., Moutou, C., Vidal-Madjar, A., et al. 2009, A\& A, 495, 959

Boisse, I., Bouchy, F., Hébrard, G., et al. 2011, A\&A, 528, A4

Boisse, I., Pepe, F., Perrier, C., et al. 2012a, $A \& A$, 545, 55

Boisse, I., Bonfils, X., \& Santos, N. C. 2012b, A\&A A, 545, 109

Bonfils, X., Mayor, M., Delfosse, X., et al. 2007, A\&A, 474, 293

Bonfils, X., Delfosse, X., Udry, S., et al. 2013, A\&A, 549, id.A109

Charbonneau et al. 2008, AAS, 212, 4402C

Czesla, S., Huber, K., Wolter, U., et al. 2009, A\& A, 505, 1277

Désert, J.-M., Charbonneau, D., Demory, B.-O., et al. 2011, ApJS, 197, 14

Desort, M., Lagrange, A;-M., Galland, F., Udry, S., \& Mayor, M. 2007, A\& A, 473, 983

Dumusque, X., Udry, S., Lovis, C., Santos, N. C., \& Monteiro, M. 2011a, A\& A, 525, 140

Dumusque, X., Santos, N. C., Udry, S., Lovis, C., \& Bonfils, X. 2011b, A\&A, 527, 82

Dumusque, X., Lovis, C., Ségransan, D., et al. 2011c, A\&A, 535, 55

Ferraz-Mello, S., Tadeu Dos Santos, M., Beaugé, C., et al. 2011, A\&A, 531, 161

Gomes da Silva, J., Santos, N. C., Bonfils, X., et al. 2012, AESA, 541, 9

Hatzes, A., Dvorak, R., Wuchterl, G., et al. 2010, A\&A, 520, 93

Huélamo, N., Figueira, P., Bonfils, X., et al. 2008, A\& A, 489, L9

Knutson, H. A., Madhusudhan, N., Cowan, N., et al. 2011, ApJ, 735, 27

Lovis, C., Dumusque, X., Santos, N. C., et al. 2011, arXiv:1107.5325

Martin, E., Guenther, E., Zapatero Osorio, M., et al. 2006, ApJ, 644, 75

Melo, C., Santos, N. C., Gieren, W., et al. 2007, A\&A, 467, 721

Moutou, C., Mayor, M., LoCurto, G., et al. 2011, A\& A, 527, 63

Nutzman, Fabrycky \& Fortney 2011, ApJ, 740, L10

Oshagh, M., Boisse, I., Boué, G., et al. 2013, A\& A, 549, id.A35

Pepe, F., Lovis, C., Ségransan, D., et al. 2011, A\&A, 534A, 58P1

Pont, F., Gilliland, R. L., Moutou, C., et al. 2007, A\&A, 476, 1347

Pont, F., Sing, D., Gibson, N., et al. 2013, MNRAS, 432, 2917

Prato, L., Huerta, M., Johns-Krull, C., et al. 2008, ApJ, 687, 103

Rabus, M., Alonso, R., Belmonte, J., et al. 2009, A\&ऽA, 494, 391

Queloz, D., Henry, G. W., Sivan, J.-P., et al. 2001, A\&A A, 379, 279

Queloz, D., Bouchy, F., Moutou, C., et al. 2009, A\&A, 506, 303

Quirrenbach, A., Amado, P. J., Seifart, W., et al. 2012, SPIE, 8446E0RQ

Sanchis-Ojeda, R., Winn, J. N., Holman, M., et al. 2011, ApJ, 733, 127

Sanchis-Ojeda \& Winn 2011, ApJ, 743, 61

Santos, N. C., Bouchy, F., Mayor, M., et al. 2004, A\& A, 426L, 19

Santos, N. C., Gomes da Silva, J., Lovis, C., \& Melo, C. 2010, A\&A, 511, 54

Ségransan, D., Mayor, M., Udry, S., et al. 2011, A\& A, 535, 54

Sing, D., Pont, F., Aigrain, S., et al. 2011, MNRAS, 416, 1443

West, A., Hawley, S., Walkowicz, L., et al. 2004, AJ, 128, 426

Wright, J. T., Marcy, G. W., Butler, R. P., et al. 2008, ApJ, 683, 63 